\title{
Correction to: Stem Cells Dev 2014;23;1937-1946
}

$\mathrm{T}$ HE AUthors of THIS ARTicle "Activation of Neural Cell Fate Programs Toward Direct Conversion of Adult Human Fibroblasts into Tri-Potent Neural Progenitors Using OCT-4," discovered during data archiving that an incorrect image representing H9 NPCs was incorporated mistakenly into panel $\mathrm{G}$ of figure 1. The correct image of human fibroblasts cultured in reprogramming media expressing $\underline{\mathrm{OCT}} 4\left(\mathrm{hFibR}^{\mathrm{OCT} 4}\right)$ cultured on poly-ornithine laminin is provided below. This does not alter the central conclusions or claims of the study in any way.

The online version of the article has been corrected to reflect these corrections.

The authors sincerely regret this error.

A Reprogramming Media Neural Progenitor Culture Neural Differentiation Culture
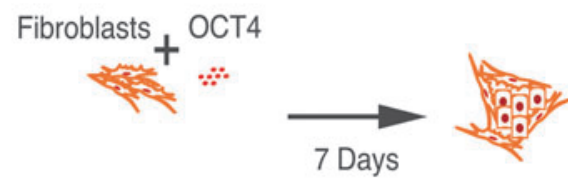

B
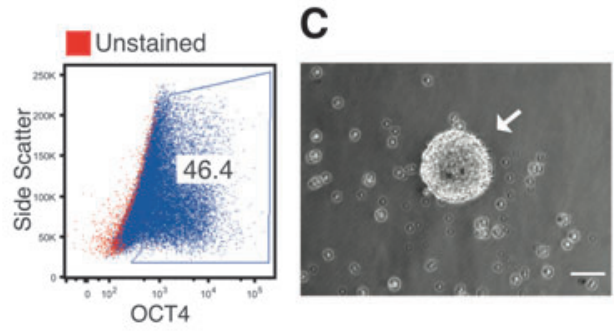

G

H
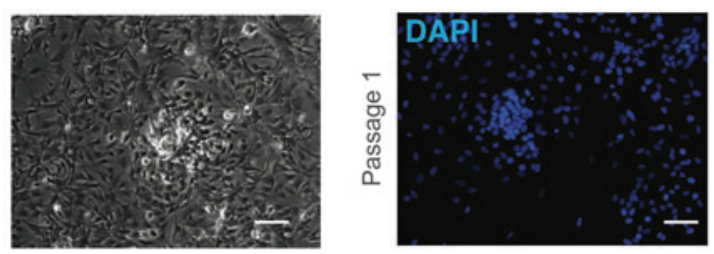
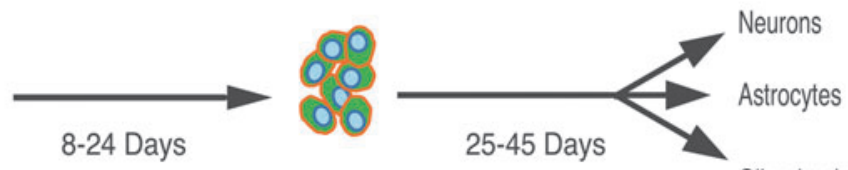

Oligodendrocytes
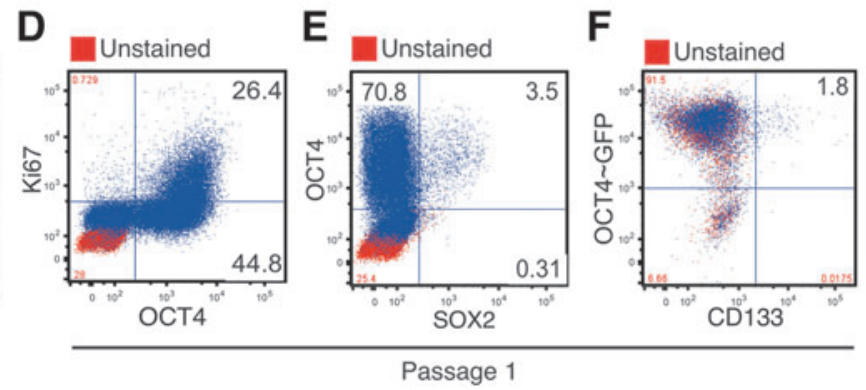

Passage 1
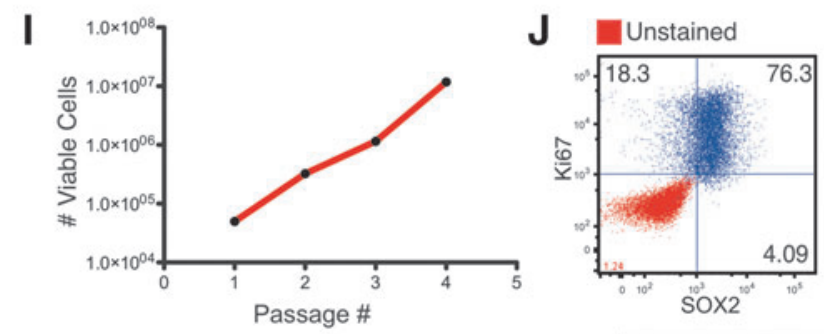

K Unstained
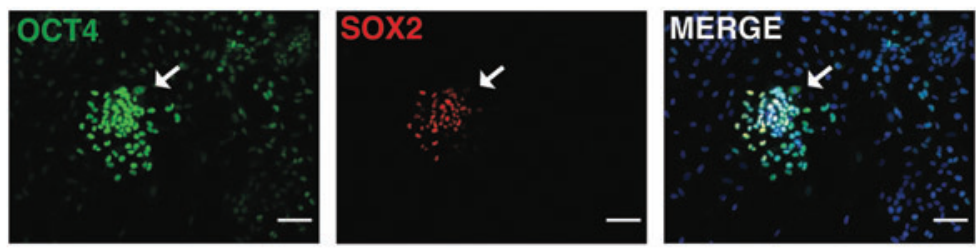

L
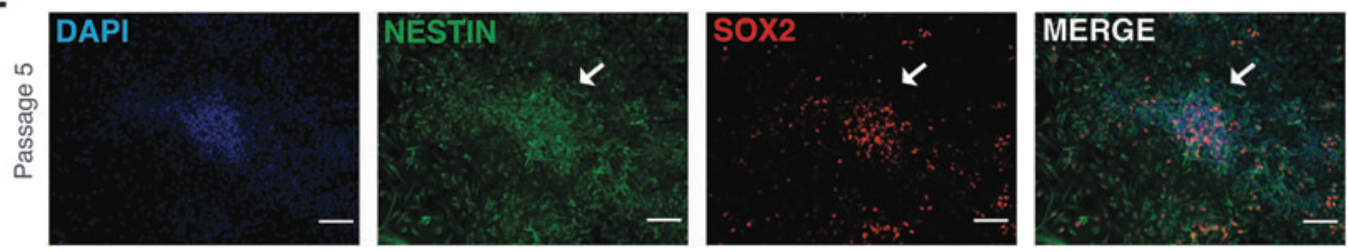